\title{
Analysis of Acoustic Cavitation Surge in a Rocket Engine Turbopump
}

\author{
Hideaki Nanri, ${ }^{1}$ Hiroki Kannan, ${ }^{1}$ Naoki Tani, ${ }^{2}$ and Yoshiki Yoshida ${ }^{1}$ \\ ${ }^{1}$ Space Transportation Propulsion Research and Development Center, Japan Aerospace Exploration Agency (JAXA), \\ 1 Takakuzo, Jinjiro, Kakuda, Miyagi 981-1526, Japan \\ ${ }^{2} J A X A$ 's Engineering Digital Innovation Center, JAXA 2-1-1, Sengen, Tsukuba, Ibaraki 305-8505, Japan
}

Correspondence should be addressed to Hideaki Nanri, nanri.hideaki@jaxa.jp

Received 25 May 2010; Revised 15 October 2010; Accepted 10 November 2010

Academic Editor: R. Amano

Copyright ( $) 2010$ Hideaki Nanri et al. This is an open access article distributed under the Creative Commons Attribution License, which permits unrestricted use, distribution, and reproduction in any medium, provided the original work is properly cited.

\begin{abstract}
In a liquid rocket engine, cavitation in an inducer of a turbopump sometimes causes instability phenomena when the inducer is operated at low inlet pressure. Cavitation surge (auto-oscillation), one such instability phenomenon, has been discussed mainly based on an inertia model assuming incompressible flow. When this model is used, the frequency of the cavitation surge decreases continuously as the inlet pressure of the turbopump decreases. However, we obtained an interesting experimental result in which the frequency of cavitation surge varied discontinuously. Therefore, we employed one-dimensional analysis based on an acoustic model in which the fluid is assumed to be compressible. The analytical result qualitatively corresponded with the experimental result.
\end{abstract}

\section{Introduction}

Launch vehicles are designed to minimize their dry mass in order to obtain better performance. As propellant tanks are major components of a rocket, decreasing their weight is a very effective way of reducing dry mass. Decreasing the pressure in a tank allows for thinner tank walls, which contributes to the efficiency of a rocket system. On the other hand, when the pressure in the tank is decreased, that is, the inlet pressure to the turbopump of the rocket engine becomes low, cavitation in the turbopump inducer sometimes causes instability phenomena. Cavitation surge, one type of cavitation instabilities, is caused not only by the unsteady characteristic of cavitation, but also by the integrated characteristics of the tank, feed pipes, valves, accumulator, and cavitation.

Cavitation surge was studied in the 1960s in relation to POGO instability, which is caused by the interaction between a rocket structure and a rocket propulsion system [1]. With regard to the characteristic of the turbopump, the dynamic transfer function of the inducer has been investigated to evaluate the instability [2]. The pump system-including a tank, pipes, a pump, and valves - has been modeled, and the stability of the system has been evaluated [3]. The several types of instabilities in turbomachines, that is, surge, rotating stall, cavitation surge, and rotating cavitation, have been examined and the unified numerical mode of these instabilities has been presented [4]. Furthermore, the frequency-response characteristics of a cavitating pump have been taken into account for modeling unstable cavitation phenomena $[5,6]$.

In these studies, however, the dynamic behavior of pressure and flow rate were evaluated with a continuity equation on the supposition that the fluid was "incompressible." The unsteady characteristics of cavitation were expressed with a mass flow gain factor $(M)$ and cavitation compliance $(K)$, then the frequency of oscillation becomes in inverse proportion to the square root of $K$ and the length of the inlet pipe $\left(1 /(\rho K L / A)^{1 / 2}\right)$ [7]. This means that when the inlet pressure decreases, cavitation compliance $(K)$ becomes large and the natural frequency of the cavitation surge decreases continuously.

However, when an experimental turbopump was tested, we found that the frequency of cavitation surge changed discontinuously as the inlet pressure decreased. This phenomenon means to be similar to acoustic resonance which has some natural frequencies [8]. In the present paper, which is aimed at explaining the discontinuity of cavitation surge frequency, we conducted simple one-dimensional analysis 


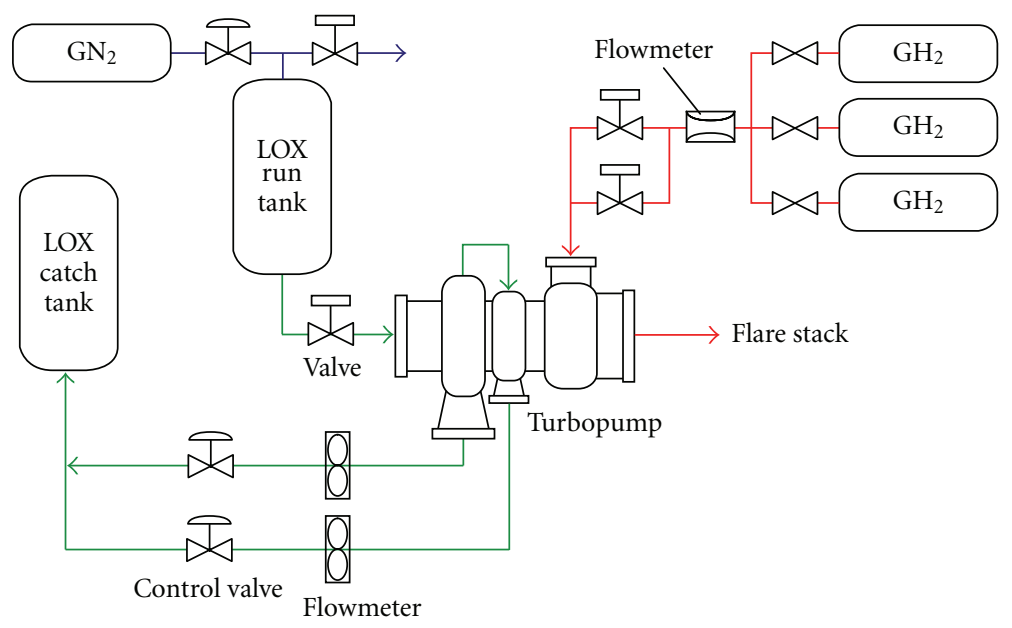

FIGURE 1: Schematic diagram of turbopump test facility.

with an acoustic model, combining the inlet pipe with the sonic velocity of liquid oxygen.

\section{Explanation of Phenomena}

Experiments on a turbopump were carried out in the highpressure liquid oxygen turbopump test facility (Figure 1) at the Kakuda Space Center of the Japan Aerospace Exploration Agency (JAXA). At this facility, the fluid is fed from a run tank of $10 \mathrm{~m}^{3}$ volume to a turbopump through a straight pipe of about $10 \mathrm{~m}$ with a valve. The fluid, discharged by the turbopump, is fed into a catch tank through pipes, flow meters, and flow control valves. The cavitation number $(\sigma)$ in the inducer can be controlled by decreasing the pressure in the run tank during a test.

Figure 2(a) shows a typical result of FFT analysis of the inlet pressure fluctuation. According to the inertia model, when the cavitation number decreases, the frequency decreases continuously, as indicated by the red line in Figure 2(b). However, the frequency of cavitation surge jumped discontinuously as the cavitation number decreased.

\section{Analytical Model}

This phenomenon mentioned above seems to be similar to acoustic resonance which has some natural frequencies. Then we assume that the fluid is "compressible" and conducted a simple one-dimensional analysis of the acoustic model. The analytical model consists of a tank, a feed pipe, and a turbopump as shown in Figure 3. We simplified the model by omitting small branches in the feed pipe and by assuming no flow fluctuation in the exit pipe of the turbopump. Although a certain capacity usually exists in the exit of pipeline, it is not taken into account in this model because the cavity volume in the turbopump has a capacity to some extent. This assumption also means that a dynamic gain of a turbopump is omitted to make this model simpler.

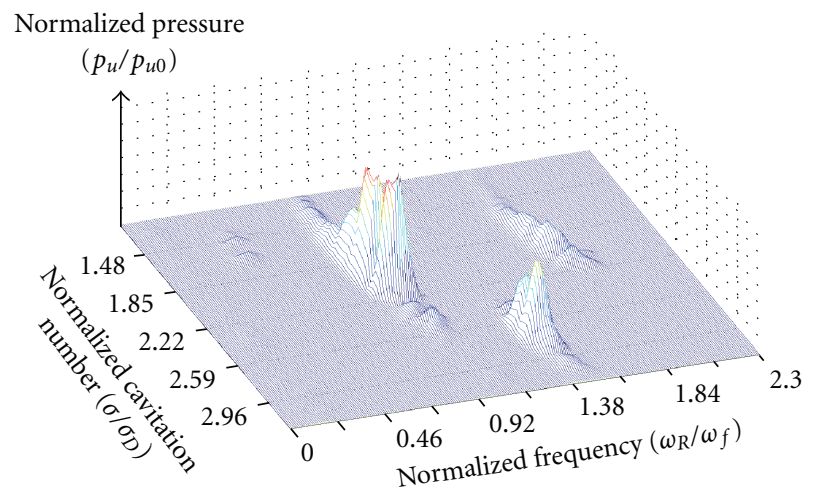

(a)

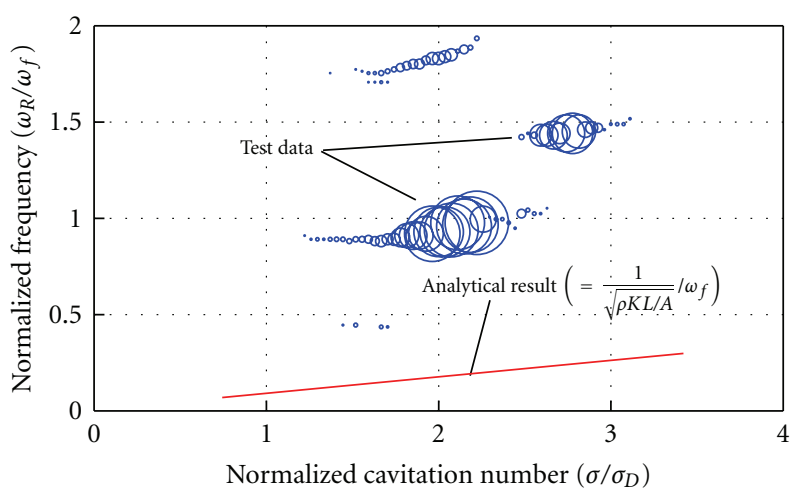

(b)

FIGURE 2: (a) FFT analyses of pressure fluctuation at inlet of the turbopump. (b) Analytical result by inertia model and experimental result of pressure fluctuation at inlet of turbopump. (The size of the bubbles indicate the amplitude of the pressure fluctuation).

Supposing that the fluid is compressible, we can write an acoustic wave equation as (1), where $K_{\text {drag }}$ is the friction coefficient defined as $\left(f_{r} U / D\right)$. The value of speed of sound 


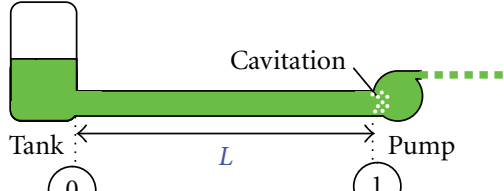

(0)

FIgURe 3: Simple analytical model.

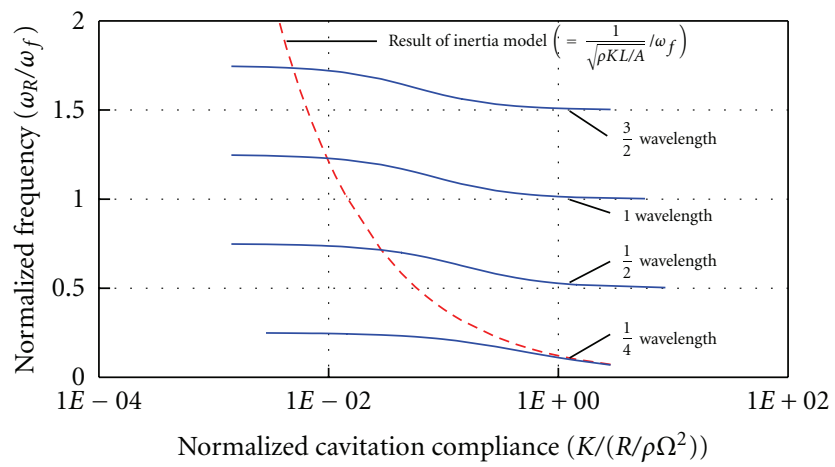

(a)

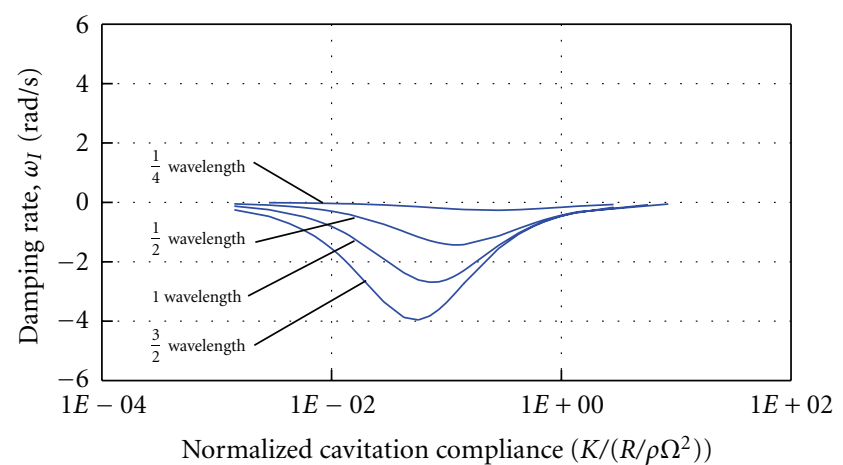

(b)

Figure 4: (a) Analytical results of the real part, $\omega_{R}$, showing trend analyses on $K\left(M \rho \Omega=0.035, K_{\text {drag }}=0\right)$. (b) Analytical results of imaginary part, $\omega_{I}$, showing trend analyses on $K(M \rho \Omega=0.035$, $\left.K_{\text {drag }}=0\right)$.

(c) is corrected with the diameter and the thickness of the pipe and its elasticity coefficient.

$$
\frac{\partial^{2} p}{\partial t^{2}}=K_{\mathrm{drag}} \frac{\partial p}{\partial t}=c^{2} \frac{\partial^{2} p}{\partial x^{2}} .
$$

For stability analyses, the pressure and the velocity are separated into steady and unsteady components: $p=p_{s}+p_{u}$ and $u=u_{s}+u_{u}$, and the absolute value of the steady part is supposed to be larger than the unsteady part $\left(p_{u} \ll p_{s}\right.$, $\left.u_{u} \ll u_{s}\right)$. And if the amplitude of pressure fluctuation at the exit of the run tank is zero, the correlation between $p_{u}$ and $u_{u}$ at the inlet of the turbopump is written as

$$
p_{u 1}=-j \rho c \frac{\sqrt{\omega^{2}-K_{\mathrm{drag}} \omega j}}{\omega} \tan \left(\frac{L}{c} \sqrt{\omega^{2}-K_{\mathrm{drag}} \omega j}\right) u_{u 1} \text {. }
$$

Furthermore, the equation of continuity at the turbopump can be written as (3) with cavitation compliance $(K)$ and mass flow gain factor $(M)$ as unsteady cavitation characteristics.

$$
\begin{gathered}
K=-\left(\frac{\partial V c}{\partial p}\right), \quad M=-\left(\frac{\partial V c}{\partial q}\right), \\
u_{u 1} A=K \dot{p}_{u 1}+M \rho A \dot{u}_{u 1} .
\end{gathered}
$$

The $p_{u 1}$ is eliminated by (2) and (3), and then the differential equation on $u_{u 1}$ is obtained.

$u_{u 1} A$

$=\left[K\left\{-j \rho c \frac{\sqrt{\omega^{2}-K_{\mathrm{drag}} \omega j}}{\omega} \tan \left(\frac{L}{c} \sqrt{\omega^{2}-K_{\mathrm{drag}} \omega j}\right)\right\}+M \rho A\right] \dot{u}_{u 1}$.

When the velocity $u_{u 1}$ is a sinusoidal oscillation, $u_{1}$ can be expressed as $e^{j \omega t}$. Then (4) is replaced by (5).

A

$$
=\left[K\left\{-j \rho c \frac{\sqrt{\omega^{2}-K_{\mathrm{drag}} \omega j}}{\omega} \tan \left(\frac{L}{a} \sqrt{\omega^{2}-K_{\mathrm{drag}} \omega j}\right)\right\}+M \rho A\right] j \omega .
$$

From this characteristic equation, the solutions of the complex frequency $\left(\omega=\omega_{R}+j \omega_{I}\right)$ can be obtained. There are many solutions because (5) has a tangential function. However, we only discuss solutions within a few harmonic overtones because the purpose of this analysis is to evaluate the experimental result as shown in Figure 2.

\section{Analytical Results and Discussion}

4.1. Effect of Cavitation Compliance $(K)$. Figure 4 shows the analytical result of the complex frequency $\left(\omega=\omega_{R}+j \omega_{I}\right)$ when cavitation compliance $(K)$ is treated as a parameter. The frequency $\left(\omega_{R}\right)$ slightly decreases along with the decrease in cavitation compliance $(K)$. This result can be explained as follows. When cavitation compliance $(K)$ is small, the inlet pipe has an open boundary condition on the run tank side and a closed boundary condition on the turbopump side. Therefore, the frequency $\left(\omega_{R}\right)$ becomes close to $(2 n+$ 1)/4 times the fundamental frequency. On the other hand, when cavitation compliance $(K)$ is large, the frequency $\left(\omega_{R}\right)$ becomes close to $n / 2$ times because both ends of the pipe have open boundary conditions. As the inlet pressure decreases, cavitation compliance $(K)$ becomes large and the boundary condition at the turbopump changes from a closed state to an open state. Consequently, the frequency of the cavitation surge $\left(\omega_{R}\right)$ slightly decreases with the decrease of the inlet pressure. In this way, when the inlet pressure of the turbopump decreases, the running turbopump performs as an intermediate boundary condition between an open and a closed state as shown in Figure 5. 


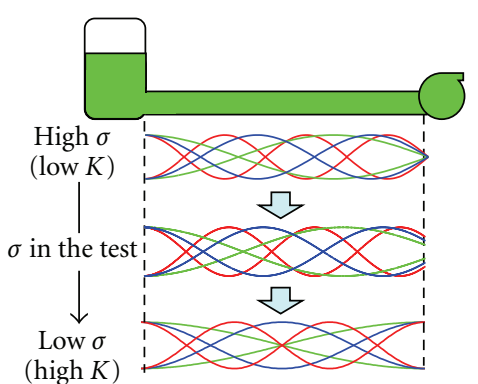

Figure 5: Acoustic mode in a feed pipe with decrease of cavitation number $(\sigma)$.

The frequency of cavitation surge is plotted as a red broken line in Figure 4(a) when it is calculated on the supposition of incompressible flow in an inertia model. As cavitation compliance $(K)$ becomes large, the frequency obtained by the inertia model becomes asymptotical to the frequency of $1 / 4$ wavelength fluctuation.

4.2. Effect of Mass Flow Gain Factor (M). Figure 6 shows the analytical result of the complex frequency $\left(\omega=\omega_{R}+j \omega_{I}\right)$ when mass flow gain factor $(M)$ is treated as a parameter. Although the frequency $\left(\omega_{R}\right)$ is not affected at all by mass flow gain factor $(M)$, the damping rate $\left(\omega_{I}\right)$ always becomes negative when mass flow gain factor $(M)$ is positive. Thus, the acoustic cavitation surge becomes unstable when mass flow gain factor $(M)$ is positive.

4.3. Effect of Coefficient of Friction in the Inlet Line ( $\left.K_{\mathrm{drag}}\right)$. Figure 7 shows the analytical result of damping rate $\left(\omega_{I}\right)$ when $K_{\text {drag }}$ is treated as a parameter. The actual value of $K_{\text {drag }}$ of the facility is about 0.6 ; however, the result in case of $K_{\text {drag }}=6$ (10 times as the actual value) is plotted in Figure 7 to make its effect more remarkable. When $K_{\text {drag }}$ increases, the value of the damping rate $\left(\omega_{I}\right)$ increases by the half number of $K_{\mathrm{drag}}$, that is, the damping rate $\left(\omega_{I}\right)$ increases by $3\left(=\left(K_{\text {drag }}=6\right) / 2\right)$ in Figure 7 . The result that the value of the damping rate $\left(\omega_{I}\right)$ increases by the half number of $K_{\mathrm{drag}}$ can be also obtained from (5) on the supposition that $\omega_{R}^{2} \gg \omega_{I}^{2}$ and $\omega_{R}^{2} \gg K_{\text {drag. }}^{2}$. Then the damping rate $\left(\omega_{I}\right)$ becomes positive, which means that the system becomes stable.

From the results of these trend analyses, we found that (1) the boundary condition on the turbopump side is incompletely open and cavitation compliance $(K)$ determines cavitation surge frequencies, (2) positive mass flow gain factor $(M)$ causes the system to be instable, and (3) friction $\left(K_{\mathrm{drag}}\right)$ makes the system more stable.

4.4. Comparisons between Analytical Results and Experimental Results. The values of cavitation compliance $(K)$ and mass flow gain factor $(M)$ of the running turbopump are necessary to calculate the analytical model. Therefore, steady state computational fluid dynamics (CFD) analyses on the inducer were conducted to obtain cavitation compliance $(K)$ and mass flow gain factor $(M)$. A cavity volume in the inducer

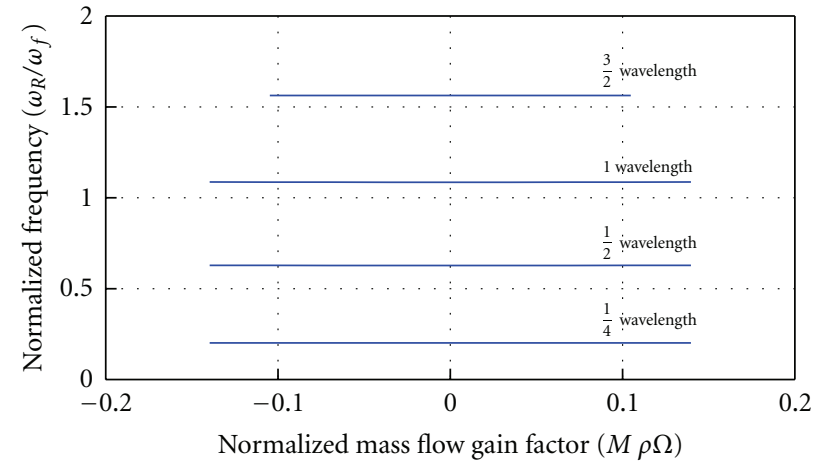

(a)

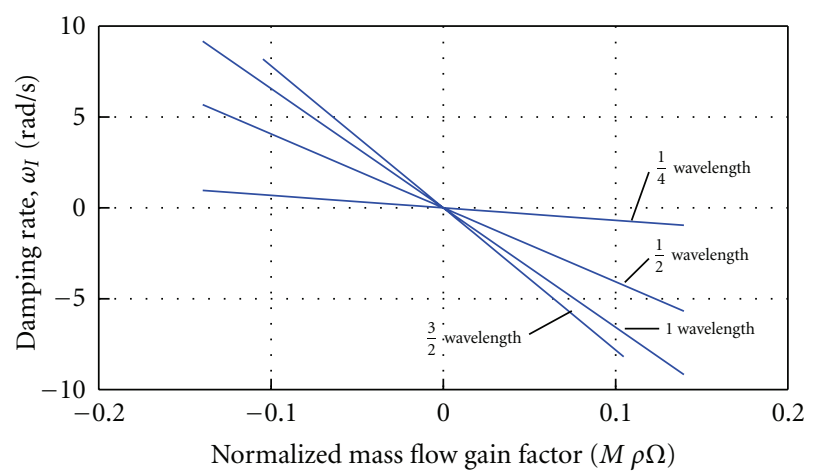

(b)

Figure 6: (a) Analytical results of real part, $\omega_{R}$, showing trend analyses on $\left(M\left(K /\left(R / \rho \Omega^{2}\right)=0.14, K_{\text {drag }}=0\right.\right.$.) (b) Analytical results of imaginary part, $\omega_{I}$, showing trend analyses on $\left(M\left(K /\left(R / \rho \Omega^{2}\right)=\right.\right.$ $\left.0.14, K_{\text {drag }}=0\right)$.

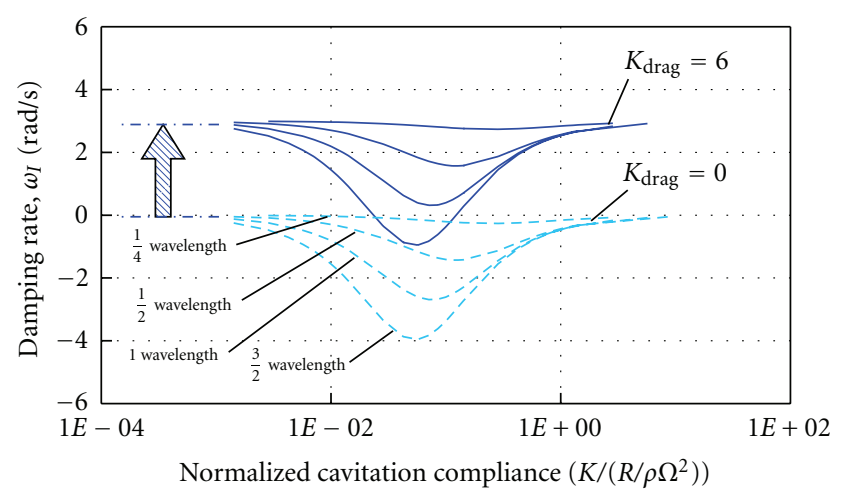

FIGURE 7: Analytical results of imaginary part, $\omega_{I}$, showing effect of $K_{\text {drag }}(M \rho \Omega=0.035)$.

under a certain inlet pressure can be obtained by steadystate CFD analysis, and another cavity volume under the different inlet pressure can be done as well. Consequently, the quasisteady value of cavitation compliance $(K)$ is estimated by these two results. The quasisteady value of mass flow gain factor $(M)$ can be estimated in the same method. This method was presented by Tani and Yamanishi [9]. 


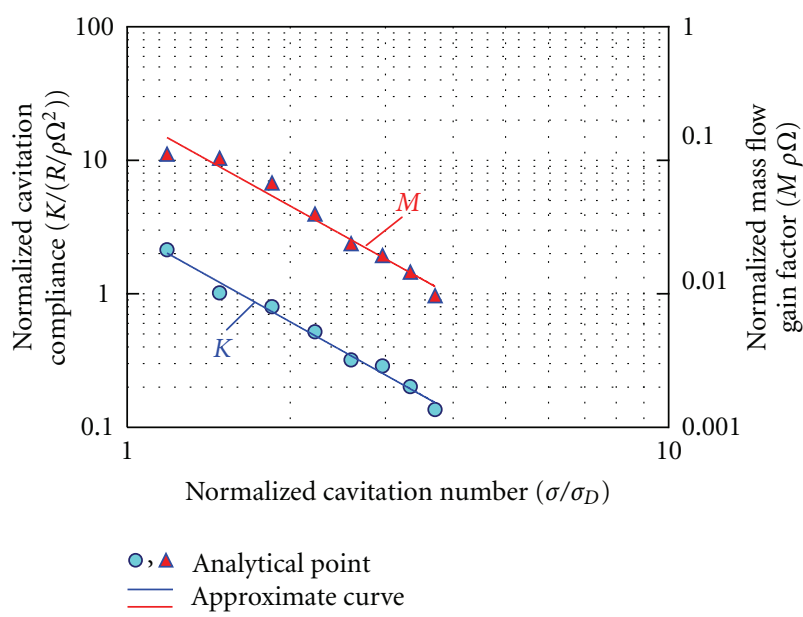

Figure 8: Cavitation characteristics ( $K$ and $M$ ) obtained with quasisteady state CFD analyses.

Figure 9(a) shows the comparison between the experimental result and analytical result using quasisteady cavitation compliance $(K)$ and mass flow gain factor $(M)$. The result of the analysis provides harmonic frequencies of acoustic resonance in the inlet pipe, and the agreement of the frequency between the experimental result and analytical result is fairly good.

Furthermore, the frequency of analytical result slightly decreases with the decrease in the cavitation number. This is the result that the boundary condition of the turbopump side changes from a closed state to an open state. The frequency of experimental result also slightly decreases as the decrease of the cavitation number, which means that the result of analysis qualitatively corresponds with the result of experiment.

All the damping rates $\left(\omega_{I}\right)$ excluding the $1 / 4$ wavelength fluctuation in higher cavitation number are negative, which means that the cavitation surge is unstable. The value of $K_{\text {drag }}$ of this facility is very small (about 0.6 ); on the other hand, those of the other test facilities are very large (the range is between 3 and 5). From the result of Figure 7, damping rates $\left(\omega_{I}\right)$ increase by the half number of $K_{\mathrm{drag}}$; then the damping rates $\left(\omega_{I}\right)$ become positive when the turbopump is tested in the other facilities. This is a result of proving that the cavitation surge was not generated in other test facilities with the same turbopump.

Nevertheless, the analysis does not express one of the experimental result that the fluctuation of $3 / 2$ wavelength disappears and that of 1 wavelength appears at the cavitation number of 2.5. One of the reasons of this phenomenon seems to be the effect of a valve equipped in the pipe; therefore, we discuss this point in the next section.

4.5. Effect of Valve Resistance in Inlet Line. A large valve is equipped in the inlet pipe between the run tank and the turbopump in the facility as shown in Figure 1, and the valve causes a local pressure drop in the inlet pipe. To evaluate the effect of the valve's resistance, we modified the analytical

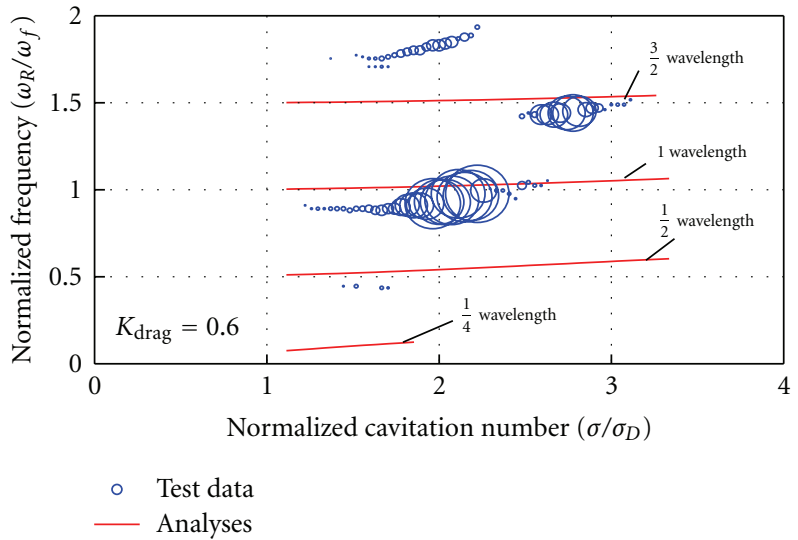

(a)

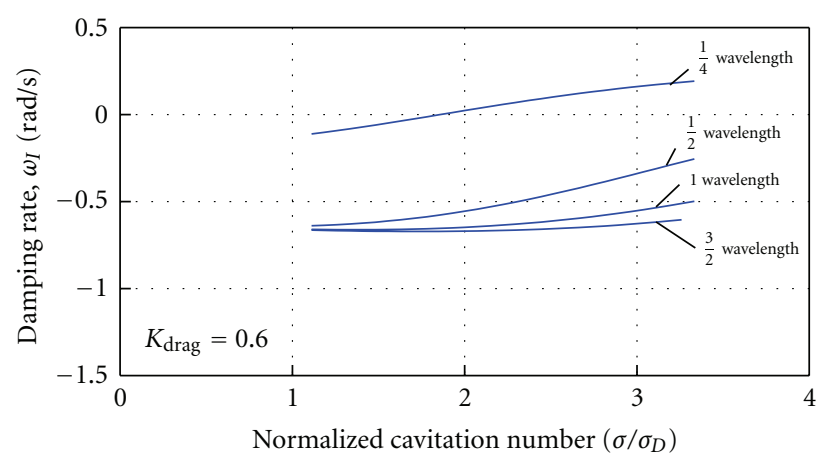

(b)

FIGURE 9: (a) Comparison of frequency between experimental results and analytical results $\left(\omega_{R}\right)$ using CFD-calculated $K$ and $M$. (b) Analytical results of damping rate $\left(\omega_{I}\right)$ using $K_{\text {drag }}$, CFDcalculated $K$ and $M$

model as shown in Figure 10. The fluid is assumed to be incompressible and the equation of motion is applied in the section between (1) and (2) in Figure 10, because the length of the valve is much smaller than that of the inlet pipe. In the other sections ((0)-(1) and (2)-( 3 ) in Figure 10), the fluid is assumed to be compressible and the wave equation is applied. In this condition, we obtain (6) at the valve inlet ((1) point in Figure 10).

$$
\begin{gathered}
p_{u 1}=-j \rho c u_{u 1} \tan \left(\frac{L_{1}}{c} \omega\right), \\
p_{u 1}-p_{u 2}=\rho L_{2} \dot{u}_{u 1}+K_{\mathrm{drag} V} \rho L_{2} u_{u 1}, \\
u_{u 1}=u_{u 2} .
\end{gathered}
$$

General solutions of pressure $\left(p_{u 2}\right)$ and velocity $\left(u_{u 2}\right)$ are obtained at the valve (at (2) point in Figure 10).

$$
\begin{aligned}
& p_{u 2}=P_{A}\left\{e^{j \omega(t-x / c)}-\frac{1-s}{1+s} e^{j \omega(t+x / c)}\right\}, \\
& u_{u 2}=\frac{P_{A}}{\rho c}\left\{e^{j \omega(t-x / c)}+\frac{1-s}{1+s} e^{j \omega(t+x / c)}\right\},
\end{aligned}
$$




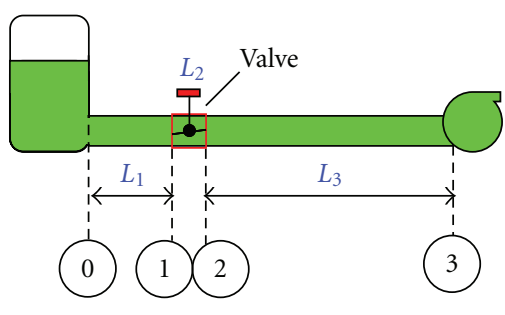

FIgURE 10: Modified analytical model with valve.

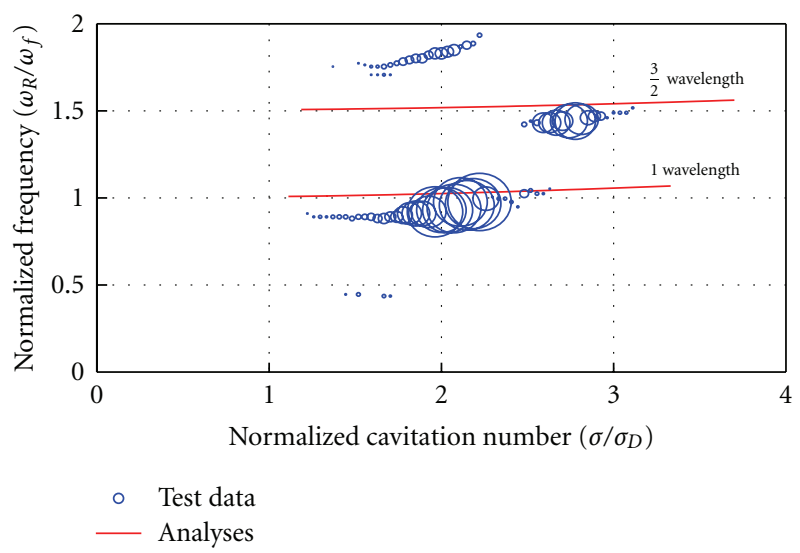

FIGURE 11: Comparison of frequency between experimental results and analytical results of the model in which valve resistance is considered, supposing $K_{\mathrm{drag}}=0, K_{\mathrm{drag} V}=125$.

where

$$
s=\frac{-j \rho c \tan \left(\left(L_{1} / c\right) \omega\right)-K_{\operatorname{drag} V} \rho L_{2}-j \rho L_{2} \omega}{\rho c} .
$$

Then, we obtain the equation that expresses the correlation between pressure and velocity in the inlet of the turbopump (at (3) point in Figure 10).

$$
\begin{gathered}
p_{u 3}=-j \rho c \tan \left(\frac{L_{3}}{c} \omega+\beta\right) u_{u 3} \\
\sin \beta=\frac{s}{\sqrt{s^{2}-1}}, \\
\cos \beta=\frac{-j}{\sqrt{s^{2}-1}} .
\end{gathered}
$$

Note that $\beta$ is a complex number. Substituting (9) into (3) yields

$$
A=\omega \rho c K \tan \left(\frac{L_{3}}{c} \omega+\beta\right)+j M \rho A \omega .
$$

In Figures 11 and 12 show the analytical result of the complex frequency $\left(\omega=\omega_{R}+j \omega_{I}\right)$ obtained by $(10)$. In Figures 11 and 12, only the results of 1 wavelength and $3 / 2$ wavelength are plotted to discuss the experimental result that the frequency changes from $3 / 2$ wavelength to 1 wavelength at the cavitation number of 2.5 .

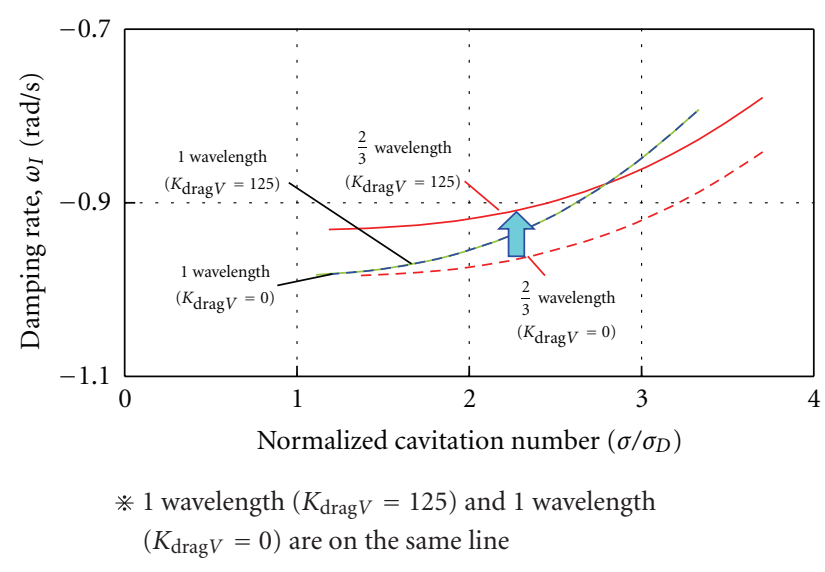

FIGURE 12: Effect of valve resistance in inlet line using CFDcalculated $K$ and $M$, supposing $K_{\text {drag }}=0$.

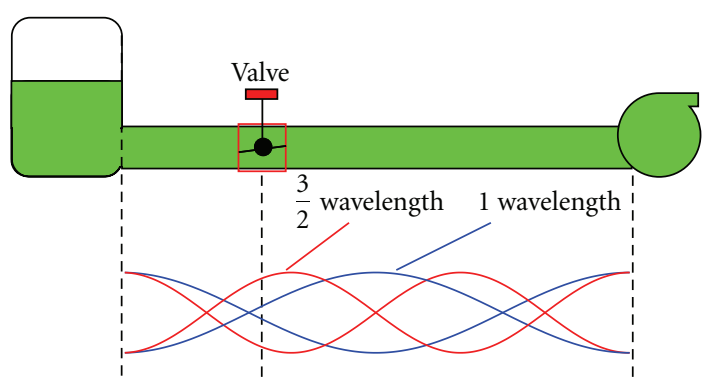

FIGURE 13: Geometric shape of acoustic modes in the feed line with the valve.

As shown in Figure 11, the frequencies of analytical result are almost the same as those of the experiments. As for the damping rate $\left(\omega_{I}\right)$ in Figure 12, the damping rate of $3 / 2$ wavelength fluctuation is increased when the effect of the valve is taken into account in this model, while that of 1 wavelength fluctuation remains almost the same.

In the inlet pipe between the tank and the turbopump, the geometrical shape of the standing wave is shown in Figure 13. The valve is located in the antinode area of $3 / 2$ wavelength fluctuation in the inlet pipe. At the same time, it is also located in the node area of 1 wavelength fluctuation. The amplitude of velocity of $3 / 2$ wavelength is much larger than that of 1 wavelength in the valve, consequently, only $3 / 2$ wavelength fluctuation is weakened.

For this reason, the damping rate of $3 / 2$ wavelength fluctuation becomes larger than that of 1 wavelength at lower cavitation numbers. This means that 1 wavelength fluctuation is more unstable than $3 / 2$ wavelength fluctuation at lower cavitation numbers. Therefore, we believe that if we obtain an exact value of cavitation compliance $(K)$ and mass flow gain factor $(M)$, this model would express the phenomenon whereby the frequency changes to a lower mode at a certain cavitation number $\left(\sigma / \sigma_{D} \sim 2.5\right)$. 


\section{Conclusion}

To explain the cavitation surge phenomenon in which frequency changes discontinuously, we conducted the onedimensional analysis by applying the acoustic model. The following points were clarified.

(1) The frequency of cavitation surge decreased discontinuously with the decrease in cavitation number in a rocket engine's turbopump test. It cannot explain the phenomenon with the current analytic model that applies the inertia model. Therefore, we took into account the factor of compressibility of the fluid and examined the acoustic model. Then, the analytical result qualitatively corresponded with the experimental result.

(2) This phenomenon is a kind of self-excited vibration caused by the coupling of the characteristic of cavitation in the turbopump and the acoustic resonance in the inlet pipe. The turbopump performs as an intermediate boundary condition between an open and a closed state because of the characteristic of the cavitation. When the inlet pressure of the turbopump decreases, the characteristic of the turbopump changes from a closed boundary condition to an open boundary condition, and then the frequency of the cavitation surge slightly decreases.

(3) The drag of the pipe acts as a damping factor of the cavitation surge, and the acoustic cavitation surge might not become unstable when the drag is large. This result is corresponding to the fact where the cavitation surge was not generated if the same turbo pump was examined by the other facilities with large pipe resistance.

(4) The local drag, generated by the valve in the pipe, weakens the fluctuation when the valve is located in the antinode of the geometrical shape of the fluctuation. Thus, the local drag becomes one of the causes of the phenomenon that the frequency of the cavitation surge changed to another frequency at a certain cavitation number.

Fitting an accumulator upstream of the turbopump is one of the effective ways to damp the fluctuation of the cavitation surge. Therefore, we will modify the analytical model to take into account the effect of the accumulator and compare the analytical result with the experimental result. In the present paper, we conducted the linear analysis, but did not discuss the nonlinear effect. In the near future, we will investigate this phenomenon by applying nonlinear methods, that is, finite element methods or a method of characteristics.

\section{Nomenclature}

A: Area of inlet pipe $\left[\mathrm{m}^{2}\right]$

c: Speed of sound $[\mathrm{m} / \mathrm{s}]$

$D$ : Diameter of inlet pipe $[\mathrm{m}]$

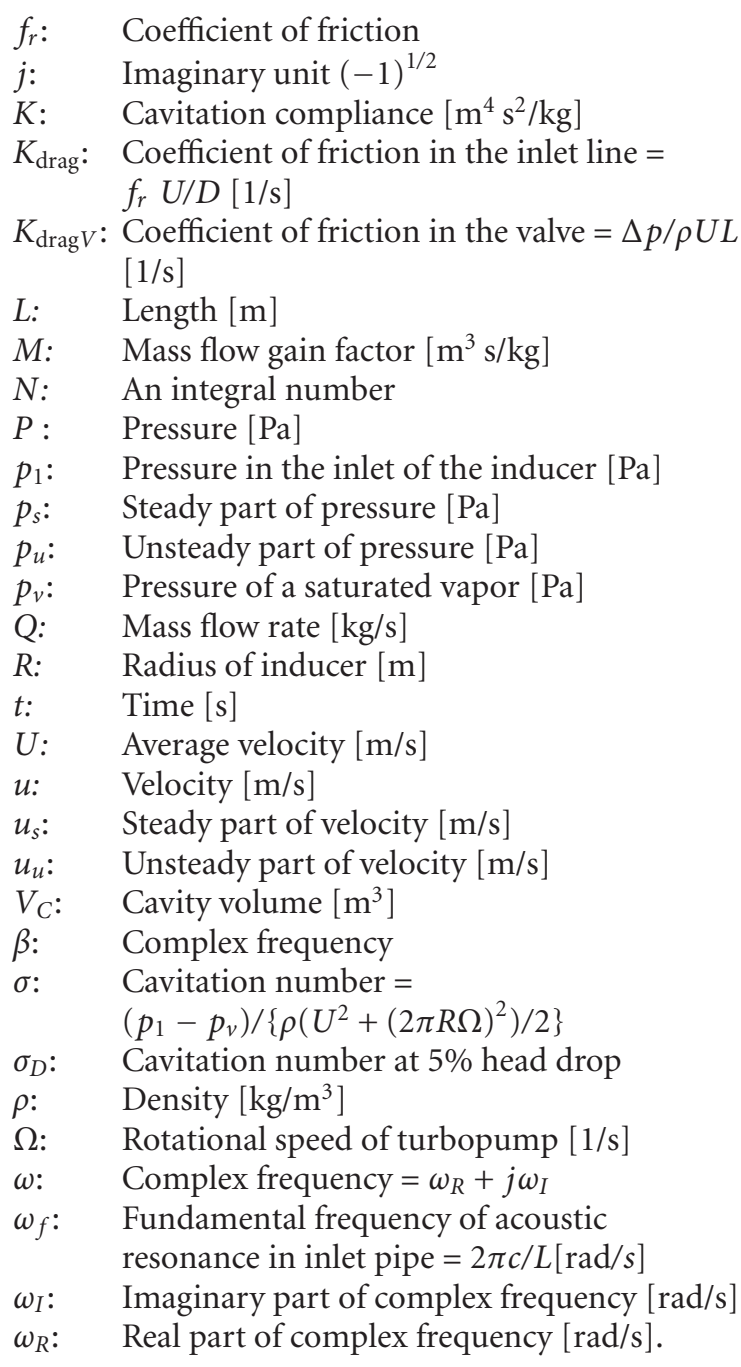

\section{Acknowledgments}

The authors wish to acknowledge the support of Mr. Koichi Okita, Mr. Syuusuke Hori, and Dr. Tomoyuki Hashimoto. They appreciate the assistance in the experiments from IHI Corporation and Mitsubishi Heavy Industries, LTD.

\section{References}

[1] S. Rubin, "Longitudinal instability of liquid rockets due to propulsion feedback (POGO)," Journal of Spacecraft and Rockets, vol. 3, no. 8, pp. 1188-1195, 1966.

[2] C. Brennen and A. J. Acosta, "The Dynamic Transfer Function for a Cavitating Inducer," ASME Journal of Fluids Engineering, vol. 98, no. 2, pp. 182-191, 1976.

[3] E. M. Greitzer, "The stability of pumping systems - the 1980 freeman scholar lecture," ASME Journal of Fluids En-gineering, vol. 103, no. 2, pp. 193-242, 1981.

[4] Y. Tsujimoto, K. Kamijo, and C. E. Brennen, "Unified treatment of flow instabilities of turbomachines," Journal of Propulsion and Power, vol. 17, no. 3, pp. 636-643, 2001.

[5] S. Rubin, "An interpretation of transfer function data for a cavitating pump," in Proceedings of the 40th AIAA Joint Propulsion Conference, Fort Lauderdale, Fla, USA, 2004, AIAA2004-4025. 
[6] X. Qiao, H. Horiguchi, and Y. Tsujimoto, "Response of backflow to flow rate fluctuations," Journal of Fluids Engineering, vol. 129, no. 3, pp. 350-358, 2007.

[7] K. Yamamoto, "Instability in a cavitating centrifugal pump," JSME International Journal 2, vol. 34, no. 1, pp. 9-17, 1991.

[8] C. Chen, C. Nicolet, K. Yonezawa et al., "One-dimensional analysis of full load draft tube surge considering the finite sound velocity in the penstock," International Journal of Fluid Machinery and Systems, vol. 2, no. 3, pp. 260-268, 2009.

[9] N. Tani and N. Yamanishi, "Cavitaion surge prediction through steady CFD," in Proceedings of the 13th Symposium on Cavitation, Sapporo, Japan, June 2006, B2-4 paper no.17. 

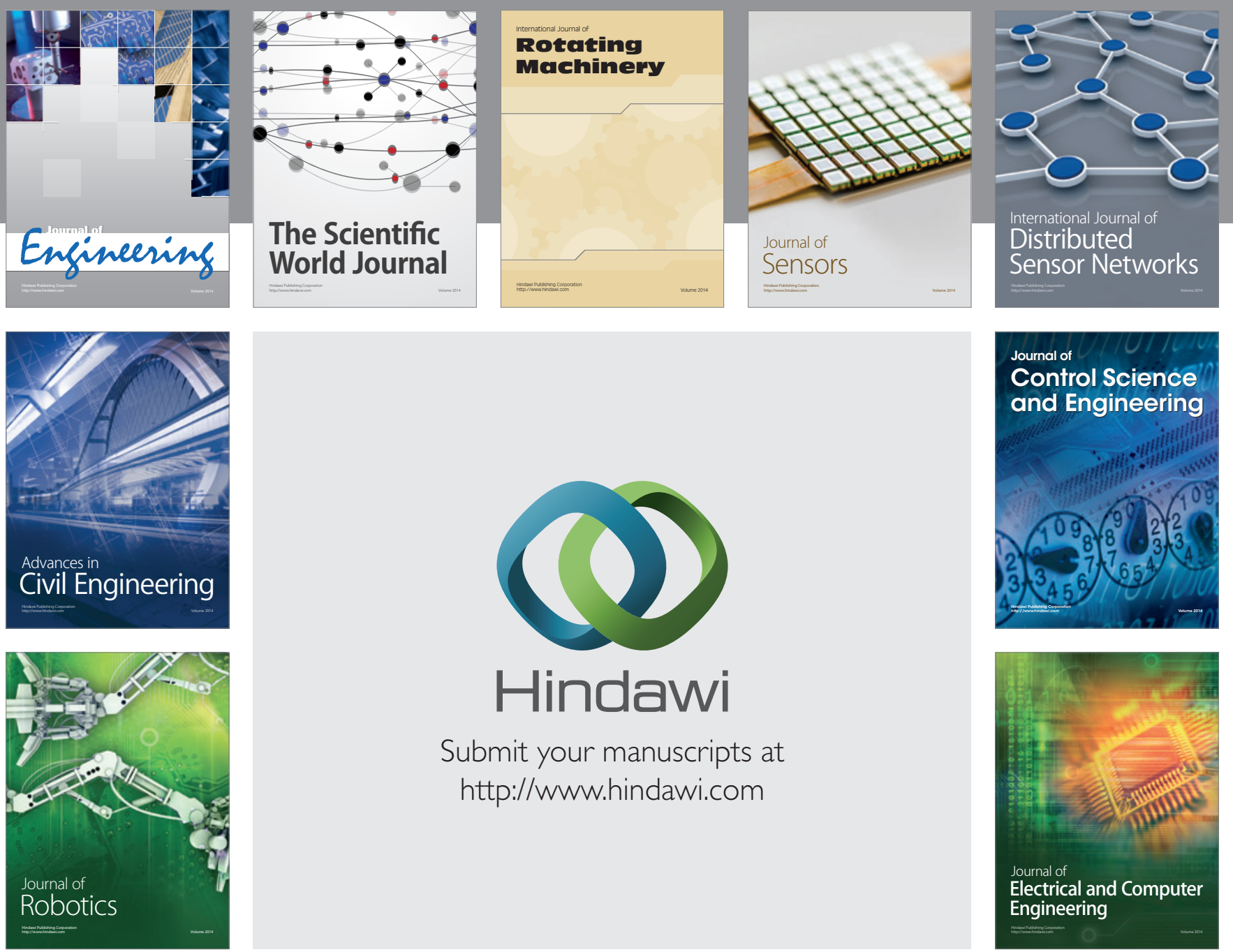

Submit your manuscripts at

http://www.hindawi.com
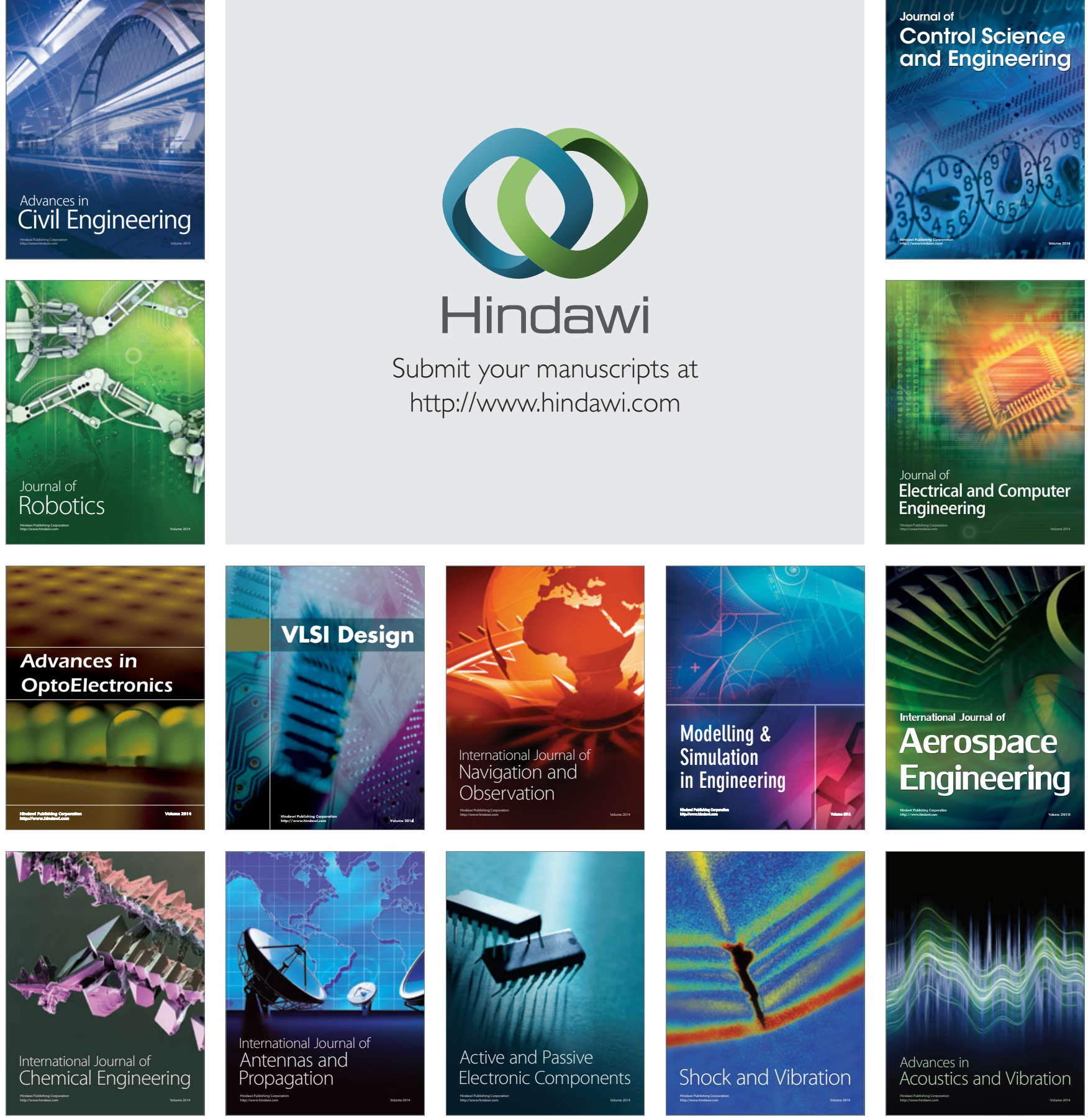